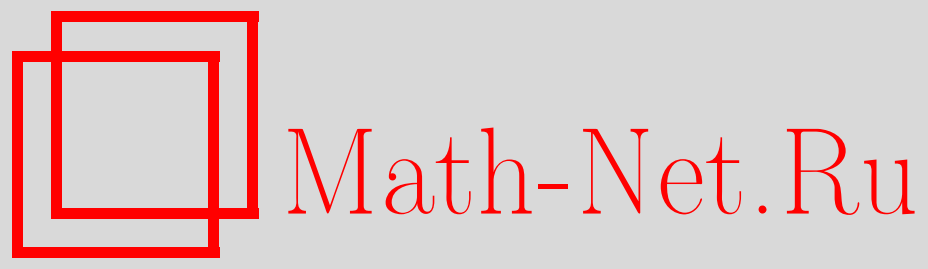

В. М. Бухштабер, Д. В. Лейкин, Решение задачи дифференцирования абелевых функций по параметрам для семейств $(n, s)$-кривых, Функи. анализ и его прил., 2008, том 42, выпуск 4, 24-36

DOI: https://doi.org/10.4213/faa2926

Использование Общероссийского математического портала Math$\mathrm{Net.Ru} \mathrm{подразумевает,} \mathrm{что} \mathrm{вы} \mathrm{прочитали} \mathrm{и} \mathrm{согласны} \mathrm{с} \mathrm{пользователь-}$ ским соглашением

http://www . mathnet.ru/rus/agreement

Параметры загрузки:

IP : 54.237 .59 .107

26 апреля 2023 г., 15:08:33

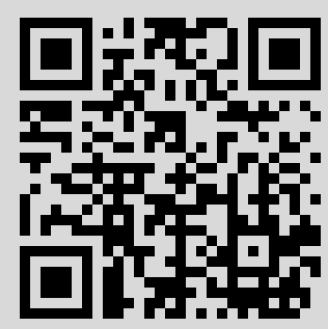




\title{
Решение задачи дифференцирования абелевых функций по параметрам для семейств $(n, s)$-кривых
}

\author{
(c) 2008. В. М. БУХштАБЕР, Д. В. ЛЕЙКИН \\ Дорогому Израилю Моисеевичу Гельфанду \\ с восхищением и глубокой благодарностью
}

\section{§1. Введение}

1.1. Постановка задачи. В классическом смысле абелевой функиией называется мероморфная функция на комплексном абелевом торе $T^{g}=\mathbb{C}^{g} / \Gamma$, где $\Gamma \subset \mathbb{C}^{g}$ - решетка ранга $2 g$. Иными словами, мероморфная функция $f$ на $\mathbb{C}^{g}$ является абелевой тогда и только тогда, когда $f(u)=f(u+\omega)$ для всех $u \in \mathbb{C}^{g}$ и $\omega \in \Gamma$. Абелевы функции на $T^{g}$ образуют дифференциальное поле.

Алгебраическая кривая $C$ рода $g$ задает решетку $\Gamma$ как множество всех периодов базисных голоморфных дифференциалов на $C$. Пусть $\boldsymbol{\omega}-$ вектор, составленный из голоморфных дифференциалов, а $\gamma-$ цикл на $C$. Тогда $\oint_{\gamma} \omega \in \Gamma$. Тор, построенный по этой решетке, называется многообразием Якоби (или якобианом) кривой $C$. Обозначим через $\mathscr{F}$ поле абелевых функций на якобиане кривой рода $g$.

Дифференциальное поле $\mathscr{F}$ обладает следующими свойствами:

(1) пусть $f \in \mathscr{F}$; тогда $\partial_{u_{i}} f \in \mathscr{F}, i=1, \ldots, g$;

(2) для любых непостоянных функций $f_{1}, \ldots, f_{g+1}$ из $\mathscr{F}$ существует такой полином $P$, что $P\left(f_{1}, \ldots, f_{g+1}\right)(u)=0$ для всех $u \in T^{g}$;

(3) пусть $f \in \mathscr{F}-$ непостоянная функция, тогда любая функция $h \in \mathscr{F}$ может быть выражена как рациональная функция от $\left(f, \partial_{u_{1}} f, \ldots, \partial_{u_{g}} f\right)$;

(4) существует целая функция $\vartheta: \mathbb{C}^{g} \rightarrow \mathbb{C}$, такая, что $\partial_{u_{i}, u_{j}} \log \vartheta \in \mathscr{F}, i, j=$ $1, \ldots, g$.

Пусть $B$ - открытое плотное подмножество в $\mathbb{C}^{d}$. Рассмотрим семейство $V$ алгебраических кривых постоянного рода $g$, гладко зависящих от параметра $b \in B$. Используя семейство $V$, зададим над $B$ пространство якобианов $U$. Пространство $U$ имеет естественную структуру гладкого многообразия и является пространством расслоения $p: U \rightarrow B$, где слоем над точкой $b \in B$ служит якобиан $J_{b}$ кривой с параметром $b$.

Обозначим через $F$ поле $C^{\infty}$-функций на $U$, ограничения которых на слои $J_{b}$ являются абелевыми функциями.

Настоящая работа посвящена следующей задаче:

(а) найти образующие $F$-модуля Der $F$ дифференцирований поля $F$;

(b) найти коммутачионные соотношения с коэфбичиентами из $F$ для образующих $F$-модуля Der $F$, m. е. описать соответствующую структуру алгебры Ли Der $F$ над полем $F$;

(c) описать действие $\operatorname{Der} F$ на $F$. 
1.2. Решение для семейства эллиптических кривых. Рассмотрим семейство плоских алгебраических кривых

$$
V=\left\{\left(x, y, g_{2}, g_{3}\right) \in \mathbb{C}^{2} \times B \mid y^{2}=4 x^{3}-g_{2} x-g_{3}\right\}
$$

над пространством параметров $B=\left\{\left(g_{2}, g_{3}\right) \in \mathbb{C}^{2} \mid \Delta \neq 0\right\}, \Delta=g_{2}^{3}-27 g_{3}^{2}$. Род $g$ кривых в этом семействе постоянен и равен 1. Такие кривые называются эллиптическими кривыми Вейерштрасса.

Слой $T^{1}$ расслоения $U$ над точкой $b=\left(g_{2}, g_{3}\right) \in B$ имеет вид $\mathbb{C}^{1} / \Gamma, \Gamma=$ $\left\{\oint_{\gamma} \frac{d x}{y}\right\}$. Здесь $\Gamma$ - решетка ранга 2 и $\gamma$ пробегает циклы на кривой $V_{b}=$ $\left\{(x, y) \in \mathbb{C}^{2} \mid y^{2}=4 x^{3}-g_{2} x-g_{3}\right\}$.

Векторное поле $\ell_{1}=\partial_{u}$ касается слоя расслоения $U$, а векторные поля $\ell_{0}=4 g_{2} \partial_{g_{2}}+6 g_{3} \partial_{g_{3}}, \ell_{2}=6 g_{3} \partial_{g_{2}}+\frac{1}{3} g_{2}^{2} \partial_{g_{3}}$ касаются дискриминанта семейства $\left\{\left(g_{2}, g_{3}\right) \in \mathbb{C}^{2} \mid \Delta=0\right\}$, поскольку $\ell_{0} \Delta=12 \Delta, \ell_{2} \Delta=0$, и задают базис векторных полей на базе $B$.

Поле послойно абелевых функций $F$ порождается координатными функциями базы $g_{2}$ и $g_{3}$ и эллиптическими функциями Вейерштрасса $\wp\left(u, g_{2}, g_{3}\right)$ и $\wp^{\prime}\left(u, g_{2}, g_{3}\right)=\partial_{u} \wp\left(u, g_{2}, g_{3}\right)$, связанными соотношением $\left(\wp^{\prime}\right)^{2}=4 \wp^{3}-g_{2} \wp-g_{3}$.

Оператор $L_{1}=\ell_{1}=\partial_{u}$, очевидно, является дифференцированием поля $F$.

Чтобы найти остальные две образующие $F$-модуля $\operatorname{Der} F$, соответствующие векторным полям $\ell_{0}, \ell_{2}$ на базе, мы используем следующие свойства сигмафункции Вейерштрасса $\sigma\left(u, g_{2}, g_{3}\right)$ - целой функции переменных $\left(u, g_{2}, g_{3}\right) \in \mathbb{C}^{3}$ :

(a) $\partial_{u}^{2} \log \sigma\left(u, g_{2}, g_{3}\right)=-\wp\left(u, g_{2}, g_{3}\right) \in F$;

(b) функция $\sigma\left(u, g_{2}, g_{3}\right)$ является решением системы линейных дифференциальных уравнений $Q_{0} \sigma=0, Q_{2} \sigma=0$, где

$$
Q_{0}=4 g_{2} \partial_{g_{2}}+6 g_{3} \partial_{g_{3}}-u \partial_{u}+1, \quad Q_{2}=6 g_{3} \partial_{g_{2}}+\frac{1}{3} g_{2}^{2} \partial_{g_{3}}-\frac{1}{2} \partial_{u}^{2}-\frac{1}{24} g_{2} u^{2} .
$$

Важным свойством операторов $Q_{0}$ и $Q_{2}$ является полиномиальная зависимость их коэффициентов от переменных $\left(u, g_{2}, g_{3}\right) \in \mathbb{C}^{3}$.

Покажем, как, используя свойства (a) и (b), получить вид операторов дифференцирования поля $F$. Начнем с уравнения $Q_{2} \sigma=0$. Имеем $Q_{2}=\ell_{2}-\frac{1}{2} \partial_{u}^{2}-$ $\frac{1}{24} g_{2} u^{2}$. Разделим $Q_{2} \sigma$ на $\sigma$ и перегруппируем слагаемые, используя функции Вейерштрасса $\zeta=\partial_{u} \log \sigma$ и $\wp=-\partial_{u}^{2} \log \sigma$. Получаем $\ell_{2} \log \sigma-\frac{1}{2} \zeta^{2}+\frac{1}{2} \wp-$ $\frac{1}{24} g_{2} u^{2}=0$. Применим $\partial_{u}^{2}$, и тогда, поскольку $\left[\partial_{u}, \ell_{2}\right]=0$, имеет место равенство $-\ell_{2} \wp+\zeta \wp^{\prime}-\wp^{2}+\frac{1}{2} \wp^{\prime \prime}-\frac{1}{12} g_{2}=0$, где $\wp^{\prime \prime}=\partial_{u}^{2} \wp$. Следовательно, $\left(\ell_{2}-\zeta \partial_{u}\right) \wp=-\wp^{2}+\frac{1}{2} \wp^{\prime \prime}-\frac{1}{12} g_{2} \in F$. Далее, так как $\left[\ell_{2}-\zeta \partial_{u}, \partial_{u}\right]=-\wp \partial_{u}$, то $\left(\ell_{2}-\zeta \partial_{u}\right) \wp^{\prime} \in F$. Действие оператора $\ell_{2}-\zeta \partial_{u}$ на функцию, зависящую только от координатных функций базы $g_{2}$ и $g_{3}$, очевидно, приводит к функции от $g_{2}$ и $g_{3}$. Таким образом, линейный дифференциальный оператор $L_{2}=\ell_{2}-\zeta \partial_{u}$, действуя на образующие поля $F$, переводит их в элементы этого поля. Следовательно, $L_{2}$ является дифференцированием поля $F$.

Аналогичное вычисление для оператора $Q_{0}$, аннулирующего сигма-функцию, приводит к еще одному дифференцированию $L_{0}=\ell_{0}-u \partial_{u}$.

Тройка образующих $F$-модуля $\operatorname{Der} F$

$$
L_{0}=-u \partial_{u}+4 g_{2} \partial_{g_{2}}+6 g_{3} \partial_{g_{3}}, \quad L_{1}=\partial_{u}, \quad L_{2}=-\zeta\left(u, g_{2}, g_{3}\right) \partial_{u}+6 g_{3} \partial_{g_{2}}+\frac{1}{3} g_{2}^{2} \partial_{g_{3}}
$$

была впервые найдена Фробениусом и Штикельбергером (см. [12]). Б. А. Дубровин разъяснил значение этого результата с точки зрения дифференциальной 
геометрии универсального расслоения якобианов эллиптических кривых. Связность в этом расслоении он назвал FS-связностью (см. [10, добавление C]). Операторы $Q_{0}$ и $Q_{2}$, аннулирующие сигма-функцию $\sigma$, были открыты Вейерштрассом (см. [14]). Во время написания работы [12] эти операторы еще не были известны, и метод Фробениуса и Штикельбергера использовал значительно более сложные вычисления, чем метод, описанный выше.

$F$-модуль $\operatorname{Der} F$ с образующими $L_{0}, L_{1}, L_{2}$ представляет собой алгебру Ли над полем $F$ с коммутационными соотношениями

$$
\left[L_{0}, L_{k}\right]=k L_{k}, \quad k=1,2, \quad\left[L_{1}, L_{2}\right]=\wp\left(u, g_{2}, g_{3}\right) L_{1} .
$$

1.3. Цель и план работы. Наша цель - выделить те случаи задачи о дифференцированиях поля абелевых функций рода $g>1$, зависящих от параметров, которые, с одной стороны, являются достаточно общими и, с другой стороны, допускают столь же эффективное решение, как и эллиптический случай. Для достижения этой цели мы

(1) выбираем специальное семейство $V \rightarrow B$ плоских алгебраических кривых постоянного рода $g$;

(2) строим базис $\ell_{1}, \ldots, \ell_{d}$ векторных полей на $B$, действующих на функции $f \in F$ как линейные дифференциальные операторы первого порядка по координатам базы $B$;

(3) строим операторы $H_{1}, \ldots, H_{d}$, действующие на $f \in F$ как операторы второго порядка вдоль слоев расслоения $U \rightarrow B$, такие, что сигма-функция $\sigma$, ассоциированная с семейством кривых $V$, удовлетворяет системе линейных дифференциальных уравнений $\left(\ell_{i}-H_{i}\right) \sigma=0, i=1, \ldots, d$.

Далее мы опишем семейства $(n, s)$-кривых и ассоциированные с ними сигмафункции $\sigma$ и, пользуясь тем же методом, что и в случае семейства эллиптических кривых, найдем образующие модуля $\operatorname{Der}(F)$, используя тот факт, что производные выше первого порядка от логарифма сигма-функции вдоль слоев расслоения $U \rightarrow B$ принадлежат полю $F$. В заключительном параграфе статьи мы опишем структуру алгебры Ли над полем $F$, соответствующую этим образующим, и приведем подробно все шаги решения задачи для семейства кривых рода 2. Часть результатов этой работы изложена в [9].

\section{$\S 2$. Семейства $(n, s)$-кривых}

2.1. Модель Вейерштрасса алгебраической кривой. Пусть $C$ - неприводимая алгебраическая кривая рода $g>0$ над $\mathbb{C}$.

Пусть $p$ - точка на $C$. Рассмотрим кольцо $M(p)$ рациональных функций на кривой $C$, полюсы которых находятся только в точке $p$. Построим последовательность $S(p)=\left(S_{1}, S_{2}, \ldots\right)$ по следующему правилу: $S_{k}=1$, если в $M(p)$ существует функция с полюсом порядка $k$, в противном случае положим $S_{k}=0$, $k \in \mathbb{N}$.

Определение 1. Точка $p$ называется регулярной, если последовательность $S(p)$ монотонна. Если последовательность $S(p)$ немонотонна, то $p$ называется точкой Вейеритрасса.

Пример 2. Пусть $p$ - регулярная точка; тогда $S(p)=(0,0, \ldots, 0,1,1,1 \ldots)$. Точки Вейерштрасса классифицируют по числу нарушений монотонности последовательности $S(p)$. Точки только с одним нарушением монотонности называются нормальными, а точки с наибольшим числом нарушений монотонности, 
равным роду кривой, называются гиперэллиптическими. Таким образом, если $p$ - нормальная точка Вейерштрасса, то $S(p)=(0,0, \ldots, 0,1,0,1,1, \ldots)$, а если $p$ - гиперэллиптическая точка Вейерштрасса, то $S(p)=(0,1,0,1, \ldots, 0,1,1, \ldots)$. Пусть $p \in C$ - точка Вейерштрасса. Обозначим через $C_{p}$ кривую $C$ с выколотой точкой $p$. Рассмотрим кольцо $\mathscr{O}$ целых рациональных функций на $C_{p}$.

Определение 3. Пусть $\phi \in \mathscr{O}$ - непостоянная функция. Полное число нулей функции $\phi$ на $C_{p}$ называется ее порядком и обозначается через ord $\phi$. Положим $\operatorname{ord} \phi=0$, когда $\phi-$ ненулевая постоянная.

Если $\phi, \varphi \in \mathscr{O}$, то $\operatorname{ord}(\phi \varphi)=\operatorname{ord} \phi+\operatorname{ord} \varphi$.

Заметим, что $\phi \in \mathscr{O}$ продолжается единственным образом до рациональной функции на $C$ с полюсом порядка ord $\phi$ в точке $p$.

Лемма 4. Пусть $\phi, \varphi \in \mathscr{O}$ - непостоянные функиии. Тогда $\varphi$ является иелой алгебраической функцией от $\phi$.

Пусть $x, y \in \mathscr{O}$ - непостоянные функции, такие, что $n=\operatorname{ord} x$ является наименьшим возможным, а $s=$ ord $y$ является наименьшим возможным при условии $\operatorname{gcd}(n, s)=1$. С помощью числового множителя функцию $у$ всегда можно нормировать так, чтобы ord $\left(y^{n}-x^{s}\right)<n s$. Тогда, поскольку у является целой алгебраической функцией от $x$, функцию $y^{n}-x^{s}$ можно представить как линейную комбинацию мономов $x^{i} y^{j}$, таких, что $\operatorname{ord}\left(x^{i} y^{j}\right)=n i+s j<n s$. Таким образом, мы получаем соотношение

$$
y^{n}-x^{s}=\sum_{i, j \geqslant 0}^{q(i, j)>0} \alpha_{i, j} x^{i} y^{j}
$$

где $q(i, j)=(n-j)(s-i)-i j$ и $\alpha_{i, j} \in \mathbb{C}$, которое задает модель Вейерштрасса кривой $C$.

Наиболее известный пример модели Вейерштрасса - это кубическое уравнение $y^{2}=4 x^{3}-g_{2} x-g_{3}$, задающее эллиптическую кривую $(n=2, s=3)$.

Вывод. Для любой неприводимой алгебраической кривой существует модель Вейерштрасса.

Замечание 5. Для кривой рода $g>1$ модель Вейерштрасса не является единственной. Модели Вейерштрасса одной кривой бирационально эквивалентны.

Следуя идее Вейерштрасса [15], вместо «кривой общего положения» мы рассматриваем классы моделей плоских алгебраических кривых

$$
V=\left\{(x, y ; \lambda) \in \mathbb{C}^{2+d} \mid y^{n}=x^{s}+\sum_{i, j \geqslant 0}^{q(i, j)>0} \lambda_{q(i, j)} x^{i} y^{j}\right\},
$$

где $d=\frac{1}{2}(n+1)(s+1)-1$ и $q(i, j)=(n-j)(s-i)-i j$, занумерованные парами целых чисел $(n, s)$, таких, что $s>n>1$ и $\operatorname{gcd}(n, s)=1$.

Род кривых, заданных моделями Вейерштрасса из $(n, s)$-класса, не превышает $g=\frac{1}{2}(n-1)(s-1)$. Например, для гиперэллиптических кривых рода не выше $g$ имеем $(n, s)=(2,2 g+1)$.

2.2. Модель Вейерштрасса с точки зрения теории особенностей. Здесь нам потребуются некоторые понятия и результаты теории особенностей [2]. 
В теории особенностей множество нулей функции вида

$$
f(x, y, \lambda)=y^{n}-x^{s}-\sum_{j=0}^{n-2} \sum_{i=0}^{s-2} \lambda_{q(i, j)} x^{i} y^{j}
$$

возникает как миниверсальная деформация так называемой особенности Фама $y^{n}-x^{s}=0$. Миниверсальная деформация зависит от $(n-1)(s-1)$ координат вектора $\lambda=\left(\lambda_{q(0,0)}, \ldots, \lambda_{q(s-2, n-2)}\right)$. Число $m=\#\left\{\lambda_{k} \mid k<0\right\}$ называется модальностъю особенности $y^{n}-x^{s}=0$.

Дискриминант $\Sigma \subset \mathbb{C}^{2 g}$ миниверсальной деформации $f$ определяется следующим образом:

$$
(\lambda \in \Sigma) \Longleftrightarrow\left(\exists(x, y) \in \mathbb{C}^{2}: f=f_{x}=f_{y}=0 \text { в точке }(x, y, \lambda)\right) .
$$

Следовательно, если $\lambda \notin \Sigma$, то род кривой из семейства, заданного миниверсальной деформацией $(1)$, не менъше $\frac{1}{2}(n-1)(s-1)$. (Напомним, что род кривой, заданной моделью Вейерштрасса из $(n, s)$-класса, не больше $\frac{1}{2}(n-1)(s-1)$.)

Наложим условие $\lambda_{q(i, j)}=0$, когда $q(i, j)<0$, на миниверсальную деформацию или, эквивалентно, условие $\lambda_{q(s-1, j)}=\lambda_{q(i, n-1)}=0$ на модель Вейерштрасса. Тогда над множеством $B=\mathbb{C}^{2 g-m} \cap\left(\mathbb{C}^{2 g} \backslash \Sigma\right)$ мы получим семейство кривых постоянного рода $g=(n-1)(s-1) / 2$ как пересечение классов миниверсальных деформаций и моделей Вейерштрасса. Такое семейство плоских алгебраических кривых мы будем называть семейством $(n, s)$-кривых.

Ниже мы будем рассматривать семейство $(n, s)$-кривых $V=\left\{(x, y ; \lambda) \in \mathbb{C}^{2} \times\right.$ $B \mid f(x, y, \lambda)=0\}$, заданное с помощью полинома

$$
f(x, y, \lambda)=y^{n}-x^{s}-\sum_{\substack{0 \leqslant i<s-1 \\ 0 \leqslant j<n-1}}^{q(i, j)>0} \lambda_{q(i, j)} x^{i} y^{j}, \quad \text { где } q(i, j)=(n-j)(s-i)-i j .
$$

Здесь $\operatorname{dim}_{\mathbb{C}} B=d=2 g-m$.

Отметим некоторые свойства семейства $(n, s)$-кривых. Многоугольник Ньютона полинома $f$ относительно переменных $(x, y)$ представляет собой треугольник. Полином $f$ однороден относительно градуировки $\operatorname{deg} x=n, \operatorname{deg} y=s$ и $\operatorname{deg} \lambda_{k}=k$. Кривая общего положения из семейства $V$, рассматриваемая как $n$-листное накрытие над $S^{2}$, имеет $(n-1) s+1=2 g+n$ точек ветвления, причем точка в бесконечности имеет число ветвления $n-1$, а числа ветвления остальных $2 g+n-1$ точек ветвления, согласно формуле Римана-Гурвица, равны 1.

2.3. Дискриминант семейства $(\boldsymbol{n}, \boldsymbol{s})$-кривых. Обозначим через $M(x, y)=$ $\left(M_{1}(x, y), \ldots, M_{2 g}(x, y)\right)$ упорядоченный по возрастанию весов набор мономов $\left\{x^{i} y^{j}\right\}, i=1, \ldots, s-2, j=1, \ldots, n-2$.

Пример 6. Пусть $(n, s)=(2,2 g+1)$; тогда $M(x, y)=\left(1, x, \ldots, x^{2 g-1}\right)$.

Пусть $(n, s)=(3,5)$; тогда $M(x, y)=\left(1, x, y, x^{2}, x y, x^{3}, y x^{2}, x^{4}\right)$.

Дискриминант миниверсальной деформации (1) и векторные поля, касательные к нему, можно эффективно построить, применив теорему Закалюкина [16]. Пусть полином $f(x, y, \lambda)$ задан формулой $(1)$. Соотношение

$$
M_{i}(x, y) f(x, y, \lambda)=\sum_{j=1}^{2 g} T_{i, j}(\lambda) M_{j}(x, y) \quad \bmod \left(\partial_{x}, \partial_{y}\right) f(x, y, \lambda)
$$


однозначно задает голоморфные функции $T_{i, j}(\lambda), i, j=1, \ldots, 2 g$. Обозначим $(2 g \times 2 g)$-матрицу, составленную из этих функций, через $T(\lambda)$.

Теорема 7. Дискриминант $\Sigma \subset \mathbb{C}^{2 g}$ полинома $f(x, y, \lambda)$, заданного формулой (1), представляет собой множество нулей голоморфной функиии $\Delta(\lambda)=$ $\operatorname{det} T(\lambda), \Sigma=\left\{\lambda \in \mathbb{C}^{2 g} \mid \Delta(\lambda)=0\right\}$. Пусть $I=\left(i_{1}, \ldots, i_{2 g}\right)-$ упорядоченный по возрастанию набор весов $\operatorname{deg} \lambda$. Векторные поля $\ell_{j}=\sum_{k=1}^{2 g} T_{2 g-j, 2 g-k}(\lambda) \partial / \partial \lambda_{i_{k}}$, $j=1, \ldots, 2 g$, касаются гиперповерхности $\Sigma$, m.е. $\ell_{j} \log \Delta(\lambda) \in \mathbb{C}[[\lambda]]$, причем $\ell_{1}=\sum_{k=1}^{2 g} i_{k} \lambda_{i_{k}} \partial / \partial \lambda_{i_{k}}$ - эйлерово векторное поле.

Составляя из векторных полей $\ell_{i}$ линейные комбинации с голоморфными по $\lambda$ коэффициентами, голоморфный репер $\mathscr{L}=\left(\ell_{1}, \ldots, \ell_{2 g}\right)^{t}$ можно привести к специальному виду, когда матрица коэффициентов репера становится симметрической (см. [7]). Сохраним для нее то же обозначение $T(\lambda)$. В этом случае $T(\lambda)$ представляет собой матрицу Арнольда (см. [1], [13]) сворачивания инвариантов особенности Фама $y^{n}-x^{s}=0$. Далее мы будем предполагать, что такая симметризация репера $\mathscr{L}$ выполнена.

Перейдем к семействам $(n, s)$-кривых. Нам необходимо построить векторные поля, касательные к $\Sigma_{0}=\Sigma \cap H$, где $H=\left\{\lambda \in \mathbb{C}^{2 g} \mid\left(\lambda_{i_{1}}, \ldots, \lambda_{i_{m}}\right)=0\right\}$. Ясно, что касательное расслоение $T \Sigma_{0}$ состоит из тех векторных полей, принадлежащих $T \Sigma$, которые ортогональны $T H$ над $H$, т. е. во всех точках, где $\left(\lambda_{i_{1}}, \ldots, \lambda_{i_{m}}\right)=0$. Рассмотрим подробнее случаи $m=0$ и $m=1$.

Если $m=0$ (случай так называемых простых особенностей), то $\Sigma_{0}=\Sigma$, и поэтому в качестве базиса в $T B$ можно использовать репер $\mathscr{L}=\left(\ell_{1}, \ldots, \ell_{2 g}\right)^{t}$. В этом случае коэффициенты репера являются полиномами от $\lambda$.

Если $m=1$, то пространство $T H$ имеет одну образующую $\eta_{1}=\partial / \partial \lambda_{i_{1}}$. Эйлерово векторное поле $\ell_{1}$, очевидно, ортогонально полю $\eta_{1}$ в $H$. Далее, для любой пары $(j, k)$, такой, что $1<j<k \leqslant 2 g$, векторное поле $\xi_{j, k}=T_{k, 1}(\lambda) \ell_{j}-T_{j, 1}(\lambda) \ell_{k}$ касается $\Sigma$ и ортогонально $\eta_{1}$ в $H$. Векторные поля $\xi_{j, k}$ удовлетворяют соотношениям $T_{l, 1}(\lambda) \xi_{j, k}-T_{k, 1}(\lambda) \xi_{j, l}+T_{j, 1}(\lambda) \xi_{k, l}=0,1<j<k<l \leqslant 2 g$. Следовательно, в этом случае для наших целей в качестве базиса в $T B$ можно использовать репер, составленный из $2 g-1$ векторных полей $\left(\ell_{1}, \xi_{2,3} \ldots, \xi_{2,2}\right)^{t}$. При ограничении на подпространство $H$ коэффициенты репера становятся полиномами от $\lambda$.

Аналогичное построение для произвольного $m$ приводит к набору из $2 g-m$ полиномиальных векторных полей. В дальнейшем репер пространства $T B$, составленный из них, мы будем обозначать через $\mathscr{L}=\left(\ell_{1}, \ldots, \ell_{2 g-m}\right)^{t}$. Обозначим через $c_{i j}^{h}(\lambda)$ структурные функции репера $\mathscr{L}$, т. е.

$$
\left[\ell_{i}, \ell_{j}\right]=\sum_{h=1}^{2 g-m} c_{i j}^{h}(\lambda) \ell_{h}, \quad i, j=1, \ldots, 2 g-m .
$$

При $m=0$ функции $c_{i j}^{h}(\lambda)$ являются полиномами. Свойства структурных функций при $m \neq 0$ мы опишем в одной из наших ближайших публикаций.

\section{§3. Сигма-функция, ассоциированная с семейством $(n, s)$-кривых}

3.1. Связность Гаусса-Манина на расслоении $(n, s)$-кривых с выколотой точкой в бесконечности. Уравнение $f(x, y, \lambda)=0$ в $\mathbb{C}^{2+2 g-m}$ задает семейство $V(n, s)$-кривых над $B=\mathbb{C}^{2 g-m} \backslash \Sigma_{0}$. Рассмотрим расслоение 
$\stackrel{\circ}{p}: \stackrel{\circ}{V} \rightarrow B$ со слоем $\stackrel{\circ}{V}_{b}$, представляющим собой кривую $V_{b}$ c выколотой точкой в бесконечности.

Пусть $H^{1}(\stackrel{\circ}{V}, \mathbb{C})$ - линейное $2 g$-мерное пространство голоморфных 1 -форм на $\stackrel{\circ}{V_{b}}$.

Рассмотрим ассоциированное с расслоением $\stackrel{\circ}{p}: \stackrel{\circ}{V} \rightarrow B$ локально тривиальное расслоение $\varpi: \Omega^{1} \rightarrow B$, слоем которого является $H^{1}(\stackrel{\circ}{V}, \mathbb{C})$. Связность в $\Omega^{1}$ называется связностъю Гаусса-Манина на $\stackrel{\circ}{\mathrm{V}}$.

Поскольку точка $\infty$ принадлежит всем кривым семейства $V$, мы можем построить глобальное сечение расслоения $\Omega^{1}$, выбрав на $V$ классический базис абелевых дифференциалов первого и второго типов.

Пусть $D(x, y, \lambda)=\left(D_{1}(x, y, \lambda), \ldots, D_{2 g}(x, y, \lambda)\right)$ - вектор канонических 1 -форм из $H^{1}\left(\stackrel{\circ}{V}_{b}, \mathbb{C}\right)$. Его матрица периодов $\Omega$ удовлетворяет тождеству Лежандра ${ }^{1)}$

$$
\Omega^{t} J \Omega=2 \pi \imath J, \quad \text { где } J=\left(\begin{array}{cc}
0_{g} & 1_{g} \\
-1_{g} & 0_{g}
\end{array}\right) .
$$

Дифференциал $D(x, y, \lambda)$ можно построить методами классической теории абелевых дифференциалов [3].

Коэффициент Кристоффеля связности Гаусса-Манина $\Gamma_{j}=\left(\Gamma_{j, i}^{k}\right), i, k=$ $1, \ldots, 2 g$, соответствующий векторному полю на базе $\ell_{j}, j=1, \ldots, 2 g-m$, однозначно определяется тем, что голоморфная 1 -форма $\ell_{j} D(x, y, \lambda)+D(x, y, \lambda) \Gamma_{j}$ точна вдоль кривой. Здесь «вдоль кривой» означает, что переменные $x$ и $y$ связаны уравнением $f(x, y, \lambda)=0$.

Интегрируя точную форму $\ell_{j} D(x, y, \lambda)+D(x, y, \lambda) \Gamma_{j}$ по базисным циклам в слое, получаем $\ell_{j} \Omega+\Omega \Gamma_{j}=0$. Из тождества Лежандра следует, что $\operatorname{det} \Omega \neq 0$. Таким образом, мы получаем

Следствие 8. $\Gamma_{j}=-\Omega^{-1} \ell_{j} \Omega$.

Применение векторного поля $\ell_{j}$ к тождеству Лежандра дает

Следствие 9. $J \Gamma_{j}+\Gamma_{j}^{t} J=0$.

Этот результат дает возможность непосредственно построить канонический дифференциал $D(x, y, \lambda)$, не используя классических методов. Положим $R(x, y, \lambda)=M(x, y) d x / f_{y}(x, y, \lambda)$. Дифференциал $R(x, y, \lambda)$, очевидно, задает базис в $\Omega^{1}$, однако его периоды не удовлетворяют тождеству Лежандра. Пусть $\chi_{j}(\lambda)$ - коэффициент Кристоффеля связности Гаусса-Манина для $R(x, y, \lambda)$ вдоль поля $\ell_{j}$, т. е. 1 -форма $\ell_{j} R(x, y, \lambda)+R(x, y, \lambda) \chi_{j}$ точна вдоль слоев расслоения $\stackrel{\circ}{p}: \stackrel{\circ}{V} \rightarrow B$. Дифференциалы $D(x, y, \lambda)$ и $R(x, y, \lambda)$ связаны соотношением $D(x, y, \lambda)=R(x, y, \lambda) K(\lambda)$, где $K(\lambda)$ - невырожденная голоморфная матрица, причем $K(0)$ - диагональная числовая матрица. Имеем $\Gamma_{j}=K^{-1}\left(\chi_{j} K-\ell_{j} K\right)$. Отсюда, согласно следствию 9 , получаем для матрицы $K$ систему $2 g-m$ уравнений

$$
\left(\chi_{j} K-\ell_{j} K\right) J K^{t}+K J\left(\chi_{j} K-\ell_{j} K\right)^{t}=0, \quad j=1, \ldots, 2 g-m .
$$

1) Частный случай соотношений Римана-Ходжа. 
3.2. Сигма-функция. Рассмотрим множество $S h$ операторов $W_{a, b, c}$, действующих на функциях по формуле

$$
W_{a, b, c}(f(u))=f(u+a) \exp \left(\frac{1}{2}\langle 2 u+a, b\rangle+c \pi \imath\right),
$$

где $u, a, b \in \mathbb{C}^{g}, c \in \mathbb{C}, \imath^{2}=-1$ и $\langle\cdot, \cdot\rangle$ обозначает евклидово скалярное произведение. Будем называть $W_{a, b, c}$ оператором ковариантного сдвига. Множество $S h$ замкнуто относительно композиции,

$$
W_{a_{2}, b_{2}, c_{2}} W_{a_{1}, b_{1}, c_{1}}=W_{a_{1}+a_{2}, b_{1}+b_{2}, c_{1}+c_{2}+(2 \pi \imath)^{-1}\left(\left\langle b_{1}, a_{2}\right\rangle-\left\langle a_{1}, b_{2}\right\rangle\right)} \cdot
$$

Нам понадобится следующее представление: $\mathbb{Z}^{g} \times \mathbb{Z}^{g} \rightarrow S h,\left(n, n^{\prime}\right) \mapsto((a, b), c)$ $=\left(\left(n, n^{\prime}\right) \Omega, \phi\left(n, n^{\prime}\right)\right)$, где $\Omega=\Omega(\lambda) \in \operatorname{Mat}\left(2 g, C^{\infty}(B)\right)$, - как и выше, матрица периодов дифференциала $D(x, y, \lambda)$, удовлетворяющая тождеству Лежандра, и $\phi: \mathbb{Z}^{2 g} \rightarrow \mathbb{Z}_{2}-$ функция Арфа.

Напомним, что $\phi: \mathbb{Z}^{2 g} \rightarrow \mathbb{Z}_{2}$ называется функиией Арфа, если для всех $q_{1}$ и $q_{2}$ из $\mathbb{Z}^{2 g}$ выполняется тождество Арфа $\phi\left(q_{1}+q_{2}\right)=\phi\left(q_{1}\right)+\phi\left(q_{2}\right)+q_{1} J q_{2}^{t}$ $\bmod 2$.

Для того чтобы задать функцию Арфа $\phi$, достаточно выбрать пару $\left(\varepsilon, \varepsilon^{\prime}\right) \in$ $\mathbb{Z}^{2 g}$. Тогда

$$
\phi\left(n, n^{\prime}\right)=\left(\left\langle n+\varepsilon, n^{\prime}+\varepsilon^{\prime}\right\rangle-\left\langle\varepsilon, \varepsilon^{\prime}\right\rangle\right) \bmod 2 .
$$

Запишем матрицу $\Omega$ в блочной форме, $\Omega=\left(\begin{array}{ll}\Omega_{1,1} & \Omega_{1,2} \\ \Omega_{2,1} & \Omega_{2,2}\end{array}\right)$; тогда можно положить $W_{\Omega}^{\varepsilon, \varepsilon^{\prime}}\left(n, n^{\prime}\right)=W_{a, b, c}$, где $a=\left(n \Omega_{1,1}+n^{\prime} \Omega_{2,1}\right), b=\left(n \Omega_{1,2}+n^{\prime} \Omega_{2,2}\right)$, $c=\left\langle n+\varepsilon, n^{\prime}+\varepsilon^{\prime}\right\rangle-\left\langle\varepsilon, \varepsilon^{\prime}\right\rangle$.

Поскольку $D(x, y, \lambda)$ - вектор базисных дифференциалов, матрица $\Omega_{1,1}$ невырожденна и $\tau=\Omega_{2,1} \Omega_{1,1}^{-1}$ - симметрическая матрица, причем, как следует из тождества Лежандра, $\operatorname{Im}(\tau)$ - положительно определенная матрица.

Положим $G_{\Omega}(u)=\left|\Omega_{1,1}\right|^{-1 / 2} \exp \left(-\frac{\pi \imath}{2} u \varkappa u^{t}\right), \imath^{2}=-1$, где, согласно тождеству Лежандра, $\varkappa=\Omega_{1,1}^{-1} \Omega_{1,2}-$ симметрическая матрица. Рассмотрим функцию

$$
\sigma\left(u, \Omega ; \varepsilon, \varepsilon^{\prime}\right)=\sum_{\left(n, n^{\prime}\right) \in \mathbb{Z}^{2 g}} W_{\Omega}^{\varepsilon, \varepsilon^{\prime}}\left(n, n^{\prime}\right) G_{\Omega}(u) .
$$

Теорема 10. Функиия $\sigma\left(u, \Omega ; \varepsilon, \varepsilon^{\prime}\right)$ является иелой функиией от $u \in \mathbb{C}^{g}$.

Мероморфные функиии $\wp_{i, j}\left(u, \Omega ; \varepsilon, \varepsilon^{\prime}\right)=-\partial_{u_{i}, u_{j}} \log \sigma\left(u, \Omega ; \varepsilon, \varepsilon^{\prime}\right)$ являются абелевыми относительно решетки, порожденной строками матрии, $\Omega_{1,1} u$ $\Omega_{2,1}$, m.e. $\wp_{i, j}\left(u, \Omega ; \varepsilon, \varepsilon^{\prime}\right)=\wp_{i, j}\left(u+n \Omega_{1,1}+n^{\prime} \Omega_{2,1}, \Omega ; \varepsilon, \varepsilon^{\prime}\right),\left(n, n^{\prime}\right) \in \mathbb{Z}^{2 g}$.

Доказательство. По построению функция $\sigma\left(u, \Omega ; \varepsilon, \varepsilon^{\prime}\right)$ является собственной функцией оператора ковариантного сдвига $W_{\Omega}^{\varepsilon, \varepsilon^{\prime}}\left(n, n^{\prime}\right)$ с собственным значением +1 или -1. Следовательно, функция $\sigma\left(u, \Omega ; \varepsilon, \varepsilon^{\prime}\right) / G_{\Omega}(u)$ разлагается в ряд Фурье с не зависящими от $u$ коэффициентами. Этот ряд сходится, так как мнимая часть матрицы $\tau$ положительно определена. С другой стороны, разность $\log \sigma\left(u, \Omega ; \varepsilon, \varepsilon^{\prime}\right)-\log \sigma\left(u+n \Omega_{1,1}+n^{\prime} \Omega_{2,1}, \Omega ; \varepsilon, \varepsilon^{\prime}\right)$ представляет собой линейную функцию от $u$.

Далее мы будем предполагать, что пара $\left(\varepsilon, \varepsilon^{\prime}\right)$ выбрана равной характеристике вектора римановых констант, которую можно считать одинаковой для всех кривых из семейства $V$. 
Определение 11. Функцию $\sigma(u, \lambda)=\Delta(\lambda)^{1 / 8} \sigma\left(u, \Omega(\lambda) ; \varepsilon, \varepsilon^{\prime}\right)$ будем называть сигма-функиией, ассочиированной с семейством кривых $V$.

Ясно, что утверждение теоремы 10 остается в силе, если в ее формулировке заменить функцию $\sigma\left(u, \Omega(\lambda) ; \varepsilon, \varepsilon^{\prime}\right)$ функцией $\sigma(u, \lambda)$.

Замечание 12. Функция $\sigma(u, \lambda)$ в отличие от $\sigma\left(u, \Omega(\lambda) ; \varepsilon, \varepsilon^{\prime}\right)$ голоморфно зависит от $\lambda$. В частности, для нее существует так называемый рациональныи предел при $\lambda \rightarrow 0$. Функция $\sigma(u, 0)$ является полиномом, который полностью определяется парой чисел $(n, s)$ и представляет собой так называемый полином Шура-Вейерштрасса (см. [6]).

3.3. Линейные операторы, аннулирующие сигма-функцию. Зададим матрицы $\alpha_{j}=\left(\alpha_{j}^{k l}\right), \beta_{j}=\left(\beta_{j k}^{l}\right)$ и $\gamma_{j}=\left(\gamma_{j k l}\right)$, где $k, l=1, \ldots, g$ и $j=1, \ldots, 2 g-$ $m$, по формуле

$$
\left(\begin{array}{cc}
\alpha_{j} & \left(\beta_{j}\right)^{t} \\
\beta_{j} & \gamma_{j}
\end{array}\right)=-J \Gamma_{j}
$$

где $\Gamma_{j}-$ символ Кристоффеля связности Гаусса-Манина, и положим

$$
H_{j}=\frac{1}{2} \alpha_{j}^{k l}(\lambda) \partial_{u_{k}} \partial_{u_{l}}+\beta_{j k}^{l}(\lambda) u_{k} \partial_{u_{l}}+\frac{1}{2} \gamma_{j k l}(\lambda) u_{k} u_{l}+\frac{1}{8} \ell_{j}(\log \Delta(\lambda))+\frac{1}{2} \beta_{j k}^{k}(\lambda) .
$$

Здесь и ниже подразумевается суммирование от 1 до $g$ по повторяющимся индексам.

Теорема 13. Сигма-функиия $\sigma(u, \lambda)$ удовлетворяет системе из $2 g-m$ линейных дифференциалъных уравнений $\left(\ell_{j}-H_{j}\right) \sigma(u, \lambda)=0, j=1, \ldots, 2 g-m$.

Доказательство. Используя соотношения $\Gamma_{j}=-\Omega^{-1} \ell_{j} \Omega$, см. следствие 8 , получаем, что $\left(\ell_{j}-H_{j}\right) G_{\Omega}(u)=0$ и $\left[\ell_{j}-H_{j}, W_{\Omega}^{\varepsilon, \varepsilon^{\prime}}\left(n, n^{\prime}\right)\right]=0$ для всех $j=$ $1, \ldots, 2 g-m$ и $\left(n, n^{\prime}\right) \in \mathbb{Z}^{2 g}$. Детали этого доказательства см. в [8].

\section{§4. Дифференцирования поля $F$}

4.1. Основной результат. Теперь, опираясь на теоремы 10 и 13, мы можем применить к полям послойно абелевых функций, ассоциированных с $(n, s)$ кривыми, метод решения нашей задачи, который мы описали в разд. 1.2 для случая $n=2$ и $s=3$.

Пусть $\mathscr{L}=\left(\ell_{1}, \ldots, \ell_{2 g-m}\right)^{t}-$ репер со структурными функциями $c_{i j}^{h}$, описанный в разд. 2.3 , и $\alpha_{j}=\left(\alpha_{j}^{k l}\right), \beta_{j}=\left(\beta_{j k}^{l}\right), \gamma_{j}=\left(\gamma_{j k l}\right)$ - матрицы, описанные в разд. 3.3 .

Теорема 14. Рассматриваемая задача для семейств $(n, s)$-кривых имеет следующее решение:

(a) $F$-модуль Der $F$ порождается $3 g-m$ образующими $d_{i}=\partial_{u_{i}}, i=1, \ldots, g$, $L_{j}=\ell_{j}-\left(\alpha_{j}^{k l} \zeta_{k}(u, \lambda)+\beta_{j k}^{l} u_{k}\right) \partial_{u_{l}}, j=1, \ldots, 2 g-m$, где $\zeta_{k}(u, \lambda)=\partial_{u_{k}} \log \sigma(u, \lambda)$;

(b) структура алгебры Ли Der $F$ над полем $F$ определяется соотношениями

$$
\begin{gathered}
{\left[d_{i}, d_{k}\right]=0, \quad\left[L_{j}, d_{i}\right]=-\left(\alpha_{j}^{k q} \wp_{i q}(u, \lambda)-\beta_{j i}^{k}\right) d_{k},} \\
{\left[L_{i}, L_{j}\right]=\frac{1}{2}\left(\alpha_{i}^{k l} \alpha_{j}^{q r}-\alpha_{j}^{k l} \alpha_{i}^{q r}\right) \wp_{k l q}(u, \lambda) d_{r}+\sum_{h=1}^{2 g-m} c_{i j}^{h} L_{h} ;}
\end{gathered}
$$


(c) действие Der F на F определяется соотношениями

$$
d_{i} \lambda=0, \quad d_{i} \wp_{q r}(u, \lambda)=\wp_{i q r}(u, \lambda),
$$

$L_{j} \lambda=\ell_{j} \lambda, \quad L_{j} \wp_{q r}(u, \lambda)=\frac{1}{2} \alpha_{j}^{k l}\left(\wp_{k l q r}-2 \wp_{k q} \wp_{l r}\right)+\beta_{j q}^{k} \wp_{k r}+\beta_{j r}^{k} \wp_{k q}-\gamma_{j q r}$.

4.2. Пример 15. Случай $(n, s)=(2,5)$ соответствует семейству кривых рода 2. Имеем $V=\left\{(x, y, \lambda) \in \mathbb{C}^{6} \mid f(x, y, \lambda)=0, \Delta(\lambda) \neq 0\right\}$, где $f(x, y, \lambda)=$ $y^{2}-\left(x^{5}+\lambda_{4} x^{3}+\lambda_{6} x^{2}+\lambda_{8} x+\lambda_{10}\right)$. Полином $f(x, y, \lambda)$ однороден в градуировке $\operatorname{deg} x=2, \operatorname{deg} y=5, \operatorname{deg} \lambda_{k}=k$ и $m=0$. Дискриминант $f(x, y, \lambda)$ задан нулями полинома

$$
\begin{gathered}
\Delta(\lambda)=3125 \lambda_{10}^{4}+2\left(128 \lambda_{8}^{5}-800 \lambda_{6} \lambda_{10} \lambda_{8}^{3}+1000 \lambda_{4} \lambda_{10}^{2} \lambda_{8}^{2}+1125 \lambda_{6}^{2} \lambda_{10}^{2} \lambda_{8}-1875 \lambda_{4} \lambda_{6} \lambda_{10}^{3}\right) \\
+108 \lambda_{10} \lambda_{6}^{5}-27 \lambda_{8}^{2} \lambda_{6}^{4}-630 \lambda_{4} \lambda_{8} \lambda_{10} \lambda_{6}^{3}+144 \lambda_{4} \lambda_{8}^{3} \lambda_{6}^{2}+825 \lambda_{4}^{2} \lambda_{10}^{2} \lambda_{6}^{2}+560 \lambda_{4}^{2} \lambda_{8}^{2} \lambda_{10} \lambda_{6} \\
-128 \lambda_{4}^{2} \lambda_{8}^{4}-900 \lambda_{4}^{3} \lambda_{8} \lambda_{10}^{2}+4 \lambda_{4}^{3}\left(4 \lambda_{10} \lambda_{6}^{3}-\lambda_{8}^{2} \lambda_{6}^{2}-18 \lambda_{4} \lambda_{8} \lambda_{10} \lambda_{6}+4 \lambda_{4} \lambda_{8}^{3}+27 \lambda_{4}^{2} \lambda_{10}^{2}\right)
\end{gathered}
$$

который можно вычислить непосредственно, взяв результант по $x$ полиномов $f(0, x, \lambda)$ и $\partial_{x} f(0, x, \lambda)$.

Векторные поля $\left(\ell_{0}, \ell_{2}, \ell_{4}, \ell_{6}\right)^{t}=T(\lambda)\left(\partial_{\lambda_{4}}, \partial_{\lambda_{6}}, \partial_{\lambda_{8}}, \partial_{\lambda_{10}}\right)^{t}, \operatorname{deg} \ell_{j}=j$, заданные симметрической матрицей

$$
T=\left(\begin{array}{cccc}
4 \lambda_{4} & 6 \lambda_{6} & 8 \lambda_{8} & 10 \lambda_{10} \\
* & 8 \lambda_{8}-\frac{12}{5} \lambda_{4}^{2} & 10 \lambda_{10}-\frac{8}{5} \lambda_{4} \lambda_{6} & -\frac{4}{5} \lambda_{4} \lambda_{8} \\
* & * & 4 \lambda_{4} \lambda_{8}-\frac{12}{5} \lambda_{6}^{2} & 6 \lambda_{4} \lambda_{10}-\frac{6}{5} \lambda_{6} \lambda_{8} \\
* & * & * & 4 \lambda_{6} \lambda_{10}-\frac{8}{5} \lambda_{8}^{2}
\end{array}\right),
$$

причем $\operatorname{det} T(\lambda)=\frac{16}{5} \Delta(\lambda)$, касаются дискриминанта, поскольку

$$
\left(\ell_{0}, \ell_{2}, \ell_{4}, \ell_{6}\right) \log \Delta(\lambda)=\left(40,0,12 \lambda_{4}, 4 \lambda_{6}\right) .
$$

Структурные функции этого репера - полиномы:

$$
\begin{gathered}
{\left[\ell_{0}, \ell_{k}\right]=k \ell_{k}, \quad k=2,4,6, \quad\left[\ell_{2}, \ell_{4}\right]=2 \ell_{6}-\frac{8}{5} \lambda_{4} \ell_{2}+\frac{8}{5} \lambda_{6} \ell_{0}} \\
{\left[\ell_{2}, \ell_{6}\right]=-\frac{4}{5} \lambda_{4} \ell_{4}+\frac{4}{5} \lambda_{8} \ell_{0}, \quad\left[\ell_{4}, \ell_{6}\right]=2 \lambda_{4} \ell_{6}-\frac{6}{5} \lambda_{6} \ell_{4}+\frac{6}{5} \lambda_{8} \ell_{2}-2 \lambda_{10} \ell_{0} .}
\end{gathered}
$$

В этом случае $M(x, y)=\left(1, x, x^{2}, x^{3}\right)$ и $R(x, y)=M(x, y) \frac{d x}{2 y}$. Имеем

$$
\begin{gathered}
\chi_{2}=\left(\begin{array}{cccc}
0 & -\frac{4}{5} \lambda_{4} & 0 & \lambda_{8} \\
1 & 0 & -\frac{8}{5} \lambda_{4} & 2 \lambda_{6} \\
0 & -1 & 0 & \frac{3}{5} \lambda_{4} \\
0 & 0 & -3 & 0
\end{array}\right), \quad \chi_{4}=\left(\begin{array}{cccc}
\lambda_{4} & -\frac{6}{5} \lambda_{6} & \lambda_{8} & 2 \lambda_{10} \\
0 & -\lambda_{4} & -\frac{2}{5} \lambda_{6} & 3 \lambda_{8} \\
-1 & 0 & 0 & \frac{2}{5} \lambda_{6} \\
0 & -3 & 0 & 0
\end{array}\right), \\
\chi_{6}=\left(\begin{array}{cccc}
0 & -\frac{3}{5} \lambda_{8} & 2 \lambda_{10} & 0 \\
-\lambda_{4} & 0 & -\frac{1}{5} \lambda_{8} & 4 \lambda_{10} \\
0 & 0 & 0 & \frac{1}{5} \lambda_{8} \\
-3 & 0 & 0 & 0
\end{array}\right) .
\end{gathered}
$$


Общее решение системы уравнений на матрицу $K(\lambda)$, удовлетворяющее условиям голоморфности и однородности, имеет вид

$$
K(\lambda)=\left(\begin{array}{cccc}
1 & 0 & a \lambda_{6} & b \lambda_{4} \\
0 & 1 & (b-1) \lambda_{4} & 0 \\
0 & 0 & 0 & -1 \\
0 & 0 & -3 & 0
\end{array}\right),
$$

где $(a, b) \in \mathbb{C}^{2}-$ свободные параметры. Положим $(a, b)=(0,0)$, что соответствует классическому построению [3]. Тогда

$$
\begin{aligned}
& \Gamma_{0}=\operatorname{diag}(3,1,-3,-1), \quad \Gamma_{2}=\left(\begin{array}{cccc}
0 & -\frac{4}{5} \lambda_{4} & \frac{4}{5} \lambda_{4}^{2}-3 \lambda_{8} & 0 \\
1 & 0 & 0 & \frac{3}{5} \lambda_{4} \\
0 & 0 & 0 & -1 \\
0 & 1 & \frac{4}{5} \lambda_{4} & 0
\end{array}\right), \\
& \Gamma_{4}=\left(\begin{array}{cccc}
\lambda_{4} & -\frac{6}{5} \lambda_{6} & \frac{6}{5} \lambda_{4} \lambda_{6}-6 \lambda_{10} & -\lambda_{8} \\
0 & 0 & -\lambda_{8} & \frac{2}{5} \lambda_{6} \\
0 & 1 & -\lambda_{4} & 0 \\
1 & 0 & \frac{6}{5} \lambda_{6} & 0
\end{array}\right), \quad \Gamma_{6}=\left(\begin{array}{cccc}
0 & -\frac{3}{5} \lambda_{8} & \frac{3}{5} \lambda_{4} \lambda_{8} & -2 \lambda_{10} \\
0 & 0 & -2 \lambda_{10} & \frac{1}{5} \lambda_{8} \\
1 & 0 & 0 & 0 \\
0 & 0 & \frac{3}{5} \lambda_{8} & 0
\end{array}\right) .
\end{aligned}
$$

Положим $\operatorname{deg} u_{i}=-i$. Тогда операторы $H_{j}$ однородны и $\operatorname{deg} H_{j}=j$ :

$H_{0}=u_{1} \partial_{u_{1}}+3 u_{3} \partial_{u_{3}}-3$,

$10 H_{2}=5 \partial_{u_{1}}^{2}+10 u_{1} \partial_{u_{3}}-8 \lambda_{4} u_{3} \partial_{u_{1}}-3 \lambda_{4} u_{1}^{2}+\left(15 \lambda_{8}-4 \lambda_{4}^{2}\right) u_{3}^{2}$,

$5 H_{4}=5 \partial_{u_{1}} \partial_{u_{3}}+5 \lambda_{4} u_{3} \partial_{u_{3}}-6 \lambda_{6} u_{3} \partial_{u_{1}}-\lambda_{6} u_{1}^{2}+5 \lambda_{8} u_{1} u_{3}+3\left(5 \lambda_{10}-\lambda_{4} \lambda_{6}\right) u_{3}^{2}-5 \lambda_{4}$, $10 H_{6}=5 \partial_{u_{3}}^{2}-6 \lambda_{8} u_{3} \partial_{u_{1}}-\lambda_{8} u_{1}^{2}+20 \lambda_{10} u_{1} u_{3}-3 \lambda_{4} \lambda_{8} u_{3}^{2}-5 \lambda_{6}$.

Образующие $F$-модуля Der $F$ :

$$
\begin{gathered}
L_{0}=\ell_{0}-u_{1} \partial_{u_{1}}-3 u_{3} \partial_{u_{3}}, \quad L_{1}=\partial_{u_{1}}, \\
L_{2}=\ell_{2}-\zeta_{1} \partial_{u_{1}}-u_{1} \partial_{u_{3}}-\frac{4}{5} \lambda_{4} u_{3} \partial_{u_{1}}, \quad L_{3}=\partial_{u_{3}}, \\
L_{4}=\ell_{4}-\zeta_{3} \partial_{u_{1}}-\zeta_{1} \partial_{u_{3}}+\lambda_{4} u_{3} \partial_{u_{3}}-\frac{6}{5} \lambda_{6} u_{3} \partial_{u_{1}}, \quad L_{6}=\ell_{6}-\zeta_{3} \partial_{u_{3}}-\frac{3}{5} \lambda_{8} u_{3} \partial_{u_{1}} .
\end{gathered}
$$

Структурные соотношения в алгебре Ли Der $F$ над полем $F$ :

$$
\begin{gathered}
{\left[L_{0}, L_{k}\right]=k L_{k}, \quad k=1,2,3,4,6,} \\
{\left[L_{1}, L_{2}\right]=\wp_{1,1} L_{1}-L_{3}, \quad\left[L_{1}, L_{3}\right]=0, \quad\left[L_{1}, L_{4}\right]=\wp_{1,3} L_{1}+\wp_{1,1} L_{3},} \\
{\left[L_{1}, L_{6}\right]=\wp_{1,3} L_{3}, \quad\left[L_{2}, L_{4}\right]=2 L_{6}-\frac{1}{2} \wp_{1,1,1} L_{3}-\frac{8}{5} \lambda_{4} L_{2}+\frac{1}{2} \wp_{1,1,3} L_{1}+\frac{8}{5} \lambda_{6} L_{0},} \\
{\left[L_{2}, L_{3}\right]=-\left(\wp_{1,3}-\frac{4}{5} \lambda_{4}\right) L_{1}, \quad\left[L_{2}, L_{6}\right]=-\frac{4}{5} \lambda_{4} L_{4}-\frac{1}{2} \wp_{1,1,3} L_{3}+\frac{1}{2} \wp_{1,3,3} L_{1}+\frac{4}{5} \lambda_{8} L_{0},} \\
{\left[L_{3}, L_{4}\right]=\left(\wp_{1,3}+\lambda_{4}\right) L_{3}+\left(\wp_{3,3}-\frac{6}{5} \lambda_{6}\right) L_{1}, \quad\left[L_{3}, L_{6}\right]=\wp_{3,3} L_{3}-\frac{3}{5} \lambda_{8} L_{1},} \\
{\left[L_{4}, L_{6}\right]=2 \lambda_{4} L_{6}-\frac{6}{5} \lambda_{6} L_{4}-\frac{1}{2} \wp_{1,3,3} L_{3}+\frac{6}{5} \lambda_{8} L_{2}+\frac{1}{2} \wp_{3,3,3} L_{1}-2 \lambda_{10} L_{0} .}
\end{gathered}
$$

Выпишем формулы, задающие действие операторов $L_{j}$ на $F$. Действие на координатные функции базы определяется с помощью соотношения $\left(L_{0}, L_{1}, L_{2}, L_{3}\right.$, $\left.L_{4}, L_{6}\right) \lambda=\left(\ell_{0}, 0, \ell_{2}, 0, \ell_{4}, \ell_{6}\right) \lambda$. Согласно пп. (2) и (3) из разд. 1.1 , достаточно 
выписать формулы для $L_{j} \wp_{1,1}$ :

$$
\begin{gathered}
L_{0} \wp_{1,1}=2 \wp_{1,1}, \quad L_{1} \wp_{1,1}=\wp_{1,1,1}, \quad L_{2} \wp_{1,1}=\frac{1}{2} \wp_{1,1,1,1}-\wp_{1,1}^{2}+\wp_{1,3}+\frac{3}{5} \lambda_{4}, \\
L_{3} \wp_{1,1}=\wp_{1,1,3}, \quad L_{4} \wp_{1,1}=\wp_{1,1,1,3}-2 \wp_{1,1} \wp_{1,3}+\frac{2}{5} \lambda_{6}, \\
L_{6} \wp_{1,1}=\frac{1}{2} \wp_{1,1,3,3}-\wp_{1,3}^{2}+\frac{1}{5} \lambda_{8} .
\end{gathered}
$$

Замечание 16. Все кривые рода 2 гиперэллиптические. Универсальные расслоения якобианов гиперэллиптических кривых рода $g$ рациональны. Этот факт был открыт Б. А. Дубровиным и С. П. Новиковым в работе [11]. Слой универсального расслоения в [11] рассматривается как поверхность уровня интегралов движения $g$-го стационарного потока системы Кортевега-де Фриза, т. е. задается системой $2 g$ алгебраических уравнений в $\mathbb{C}^{3 g}$, степень которых растет с ростом рода. В работах [4], [5] предложены координаты, в которых слой задается системой $2 g$ уравнений степени не выше 3 .

Для универсального пространства кривых рода 1 координаты ДубровинаНовикова и координаты из [4], [5] совпадают. А именно, в $\mathbb{C}^{3}$ с координатами $\left(x_{2}, x_{3}, x_{4}\right), \operatorname{deg} x_{i}=i$, слой над точкой $b=\left(g_{2}, g_{3}\right) \in B$, см. разд. 1.2 , задается уравнениями $g_{2}=12 x_{2}^{2}-2 x_{4}, g_{3}=-8 x_{2}^{3}+2 x_{4} x_{2}-x_{3}^{2}$ и параметризуется эллиптическими функциями $\left(x_{2}, x_{3}, x_{4}\right)=\left(\wp(u, b), \wp^{\prime}(u, b), \wp^{\prime \prime}(u, b)\right)$, где $u$ пробегает якобиан $J_{b}-$ слой расслоения $U \rightarrow B$, см. разд. 1.1. Далее, в этих координатах $\Delta=-432 x_{2}^{3} x_{3}^{2}-27 x_{3}^{4}+108 x_{2} x_{3}^{2} x_{4}+36 x_{2}^{2} x_{4}^{2}-8 x_{4}^{3}$, а дифференцирования $L_{0}, L_{1}$ и $L_{2}$ поля $F$ представляются векторными полями с полиномиальными коэффициентами

$$
\begin{gathered}
L_{0}=2 x_{2} \partial_{x_{2}}+3 x_{3} \partial_{x_{3}}+4 x_{4} \partial_{x_{4}}, \quad L_{1}=x_{3} \partial_{x_{2}}+x_{4} \partial_{x_{3}}+12 x_{2} x_{3} \partial_{x_{4}}, \\
L_{2}=\frac{2}{3}\left(x_{4}-3 x_{2}^{2}\right) \partial_{x_{2}}+3 x_{2} x_{3} \partial_{x_{3}}+\left(3 x_{3}^{2}+2 x_{2} x_{4}\right) \partial_{x_{4}}
\end{gathered}
$$

касательными к множеству нулей функции $\Delta$.

Для универсального пространства кривых рода 2, согласно [4], [5], в $\mathbb{C}^{6}$ с координатами $\left(x_{2}, x_{3}, x_{4}, z_{4}, z_{5}, z_{6}\right), \operatorname{deg} x_{i}=i$ и $\operatorname{deg} z_{j}=j$, слой над точкой $b=\left(\lambda_{4}, \lambda_{6}, \lambda_{8}, \lambda_{10}\right) \in B$ задается уравнениями

$$
\begin{gathered}
6 x_{2}^{2}-x_{4}+4 z_{4}+2 \lambda_{4}=0, \quad 8 x_{2}^{3}-2 x_{2}\left(x_{4}+4 z_{4}\right)+x_{3}^{2}+2 z_{6}-4 \lambda_{6}=0, \\
8 x_{2}^{2} z_{4}-x_{2} z_{6}+2 z_{4}^{2}-x_{4} z_{4}+x_{3} z_{5}-2 \lambda_{8}=0, \quad 8 x_{2} z_{4}^{2}-2 z_{4} z_{6}+z_{5}^{2}-4 \lambda_{10}=0
\end{gathered}
$$

и параметризуется гиперэллиптическими абелевыми функциями

$$
\left(x_{1}, x_{2}, x_{4}\right)=\left(1, L_{1}, L_{1}^{2}\right) \wp_{1,1}(u, b), \quad\left(z_{4}, z_{5}, z_{6}\right)=\left(1, L_{1}, L_{1}^{2}\right) \wp_{1,3}(u, b),
$$

где $u=\left(u_{1}, u_{3}\right)$ пробегает якобиан $J_{b}-$ слой расслоения $U \rightarrow B$. Отметим, что найденные выше дифференцирования поля $F$, как и в случае эллиптических функций, переходят в векторные поля с полиномиальными коэффициентами, касающиеся «дискриминанта». Возникающие таким образом алгебраические динамические системы будут описаны в наших ближайших публикациях.

Координаты Дубровина-Новикова $\left(U_{2}, U_{3}, \ldots, U_{7}\right)$, где $\operatorname{deg} U_{i}=i$, выражаются через координаты $\left(x_{2}, x_{3}, x_{4}, z_{4}, z_{5}, z_{6}\right)$ по формулам

$$
\begin{gathered}
U_{2}=x_{2}, \quad U_{3}=x_{3}, \quad U_{4}=x_{4}, \quad U_{5}=4\left(3 x_{2} x_{3}+z_{5}\right), \\
U_{6}=4\left(3 x_{3}^{2}+3 x_{2} x_{4}+z_{6}\right), \quad U_{7}=4\left(36 x_{3} x_{2}^{2}+20 x_{2} z_{5}+9 x_{3} x_{4}+4 x_{3} z_{4}\right) .
\end{gathered}
$$


Этим координатам соответствует следующая параметризация слоя над точкой $b \in B$ :

$$
\left(U_{2}, U_{3}, U_{4}, U_{5}, U_{6}, U_{7}\right)=\left(1, L_{1}, L_{1}^{2}, L_{1}^{3}, L_{1}^{4}, L_{1}^{5}\right) \wp_{1,1}(u, b)
$$

\section{ЛитерАТУРА}

[1] В. И. Арнольд, Особенности каустик и волновых фронтов, ФАЗИС, Москва, 1996.

[2] В. И. Арнольд, А. Н. Варченко, С. М. Гусейн-Заде, Особенности дифферениируемых отображений, МЦНМО, М., 2004.

[3] Г. Бейкер, Абелевъ функиии, МЦНМО, М., 2008.

[4] V. M. Buchstaber, V. Z. Enolskii, D. V. Leykin, Hyperelliptic Kleinian functions and applications, in: Solitons, Geometry and Topology: On the Crossroad, Adv. Math. Sci., Amer. Math. Soc. Transl. Ser. 2, vol. 179, Amer. Math. Soc., Providence, RI, 1997, $1-34$.

[5] V. M. Buchstaber, V. Z. Enolskii, D. V. Leykin, Kleinian functions, hyperelliptic Jacobians and applications, Rev. Math. Math. Phys., 10:2 (1997), 3-120.

[6] В. М. Бухштабер, Д. В. Лейкин, В. З. Энольский, Ращионалъные аналоги абелевых функиий, Функц. анализ и его прил., 33:2 (1999), 1-15.

[7] В. М. Бухштабер, Д. В. Лейкин, Полиномиальные алгебры Ли, Функц. анализ и его прил., 36:4 (2002), 18-34.

[8] В. М. Бухштабер, Д. В. Лейкин, Уравнения теплопроводности в неголономном penepe, Функц. анализ и его прил., 38:2 (2004), 12-27.

[9] В. М. Бухштабер, Д. В. Лейкин, Дифференцирование абелевых функиий по параметрам, УМH, 62:4 (2007), 153-154.

[10] B. A. Dubrovin, Geometry of $2 D$ topological field theories, in: Lecture Notes in Math., vol. 1620, 1994, 120-348.

[11] Б. А. Дубровин, С. П. Новиков, Периодическая задача для уравнения Кортевегаде Фриза и Штурма-Лиувилля. Их связь с алгебраической геометрией, Докл. AH CCCP, 219:3 (1974), 531-534.

[12] F. G. Frobenius, L. Stickelberger, Über die Differentiation der elliptischen Functionen nach den Perioden und Invarianten, J. Reine Angew. Math., 92 (1882), 311-337.

[13] А. Б. Гивенталь, Сворачивание инвариантов групп, порожденных отражениями и связанных с простыми особенностями функиий, Функц. анализ и его прил., 14:2 (1980), 4-14.

[14] K. Weierstrass, Zur Theorie der elliptischen Funktionen, in: Mathematische Werke, Bd. 2, Teubner, Berlin, 1894, 245-255.

[15] K. Weierstrass, Abelschen Funktionen, Gesammelte Werke, Bd. 4, 1904.

[16] В. М. Закалюкин, Перестройки волновых фронтов, зависящих от одного параметра, Функц. анализ и его прил., 10:2 (1976), 69-70.

Математический институт им. В. А. Стеклова

e-mail: buchstab@mi.ras.ru

Поступило в редакцию 3 сентября 2008 г.

Институт магнетизма, Киев, Украина

e-mail: dile@imag.kiev.ua 